\title{
Rotation of Phase Difference in Four Coupled Oscillators as a Regular Tetrahedron Form
}

\author{
Takahiro Nagai, Yoko Uwate and Yoshifumi Nishio \\ Dept. of Electrical and Electronic Engineering, \\ Tokushima University \\ 2-1 Minami-Josanjima, Tokushima, Japan \\ Email: \{nagataka, uwate, nishio\}@ee.tokushima-u.ac.jp
}

\begin{abstract}
Coupled oscillatory systems are suitable to express synchronization phenomena, and it is important to unveil nonlinear phenomena. In this study, we investigate the synchronization phenomena observed in four coupled van der Pol oscillators as a regular tetrahedron form. We observe the rotation of the phase difference between anti-phase state and asynchonization state. Furthermore, the sojourn time of the anti-phase states is calculated.
\end{abstract}

\section{INTRODUCTION}

Synchronization is one of the basic natural phenomena, and it is regarded typical nonlinear phenomena. Coupled oscillatory systems are good model to express high dimensional nonlinear phenomena in the natural science fields. Therefore investigations of the coupled oscillatory systems are reported in various research fields [1]-[7]. Besides, many kind of coupled systems have been investigated to analyze the mechanism of the nonlinear phenomena. We can see that coupled oscillatory systems produce interesting phase wave patterns, including wave propagation, clustering and complex patterns. However, synchronization phenomena of the oscillators have not been analyzed enough yet. Therefore, we have to research the case of more complicated synchronization phenomena to elucidate high dimensional nonlinear phenomena.

Here, van der Pol oscillators were coupled in various form and were investigated about their synchronization phenomena by using the theoretically and the experimentally methods [8][10]. In [11], it is studied synchronization phenomena about three van der Pol oscillators coupled as a ring topology. In this circuit system, each oscillator was coupled by an

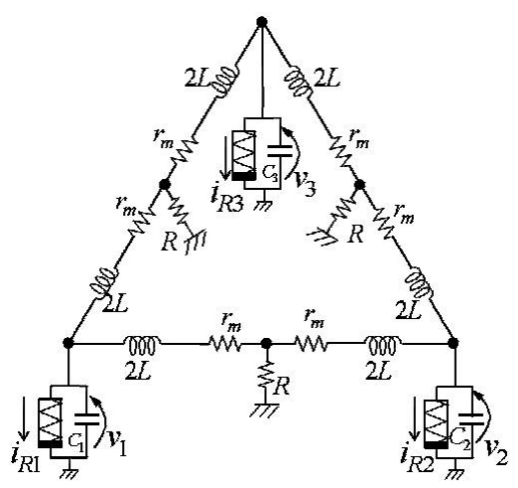

Fig. 1. Three coupled van der Pol oscillators.

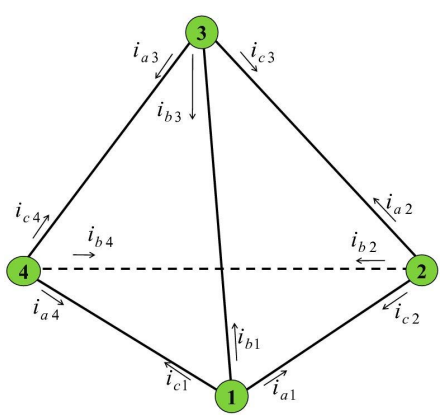

Fig. 2. Conceptual circuit model for tetrahedron form.

inductor and the number of coupled oscillators was an odd number. And then the coupled oscillators could not synchronize with in/anti-phase states. In other words, three-phase synchronization (phase shift: $120^{\circ}$ ) is obtained for the case of three coupled oscillators by the effect of frustration. However, the three-phase synchronization was always observed stably in that system. Therefore we consider that what kind of synchronization phenomena are observed by using oscillators with stronger frustration.

In this study, we investigate synchronization phenomena in coupled oscillatory system which has stronger frustrations. We consider four coupled van der Pol oscillators as the regular tetrahedron form as shown in Fig. 2. By computer simulations, we observe the rotation of phase difference between anti-phase synchronization and asynchronization periodically. Namely, the adjacent oscillators are trapped into the anti-phase state at certain time. In addition, we calculate the sojourn time of anti-phase states in one-period, when the parameters of the nonlinearity and the coupling strength are changed.

\section{Circuit Model}

The circuit model of four coupled van der Pol oscillators as the regular tetrahedron form is shown in Fig. 3. Each van der Pol oscillator is composed of an inductor, a negative resistor and a capacitor. In this study, the adjacent oscillators are coupled by resistor via inductors.

In this figure, we assume that the electric currents flowing from each top are $i_{a k}, i_{b k}$ and $i_{c k}$, the voltages of the capacitor of each oscillator are $v_{k}$ and the electric currents flowing from the negative resistance are $i_{R k}$. When we couple four oscillators, tiny resistor $r_{m}$ is introduced to consider internal 


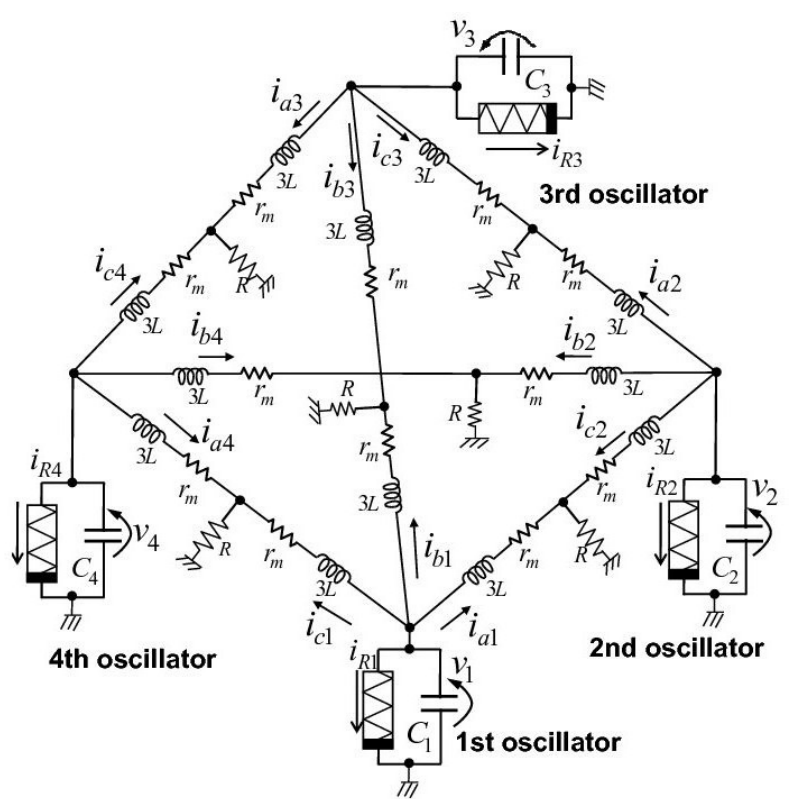

Fig. 3. Four coupled van der Pol oscillators.

resistance. In addition, we connect tiny resistor to avoid $L$ loop.

In the computer simulations, we assume that the $v_{k}-i_{R k}$ characteristics of nonlinear resistor in each oscillator is given by the following third order polynomial equation.

$$
\begin{array}{ll}
i_{R k}=-g_{1} v_{k}+g_{3} v_{k}{ }^{3} & \left(g_{1}, g_{3}>0\right), \\
& (k=1,2,3,4) .
\end{array}
$$

The normalized circuit equations are expressed as:

[First oscillator]

$$
\left\{\begin{aligned}
\frac{d x_{1}}{d \tau} & =\varepsilon\left(1-x_{1}^{2}\right) x_{1}-\left(y_{a 1}+y_{b 1}+y_{c 1}\right) \\
\frac{d y_{a 1}}{d \tau} & =\frac{1}{3}\left\{x_{1}-\eta y_{a 1}-\gamma\left(y_{a 1}+y_{c 2}\right)\right\} \\
\frac{d y_{b 1}}{d \tau} & =\frac{1}{3}\left\{x_{1}-\eta y_{b 1}-\gamma\left(y_{b 1}+y_{b 3}\right)\right\} \\
\frac{d y_{c 1}}{d \tau} & =\frac{1}{3}\left\{x_{1}-\eta y_{c 1}-\gamma\left(y_{c 1}+y_{a 4}\right)\right\}
\end{aligned}\right.
$$

[Second oscillator]

$$
\left\{\begin{aligned}
\frac{d x_{2}}{d \tau} & =\varepsilon\left(1-x_{2}{ }^{2}\right) x_{k}-\left(y_{a 2}+y_{b 2}+y_{c 2}\right) \\
\frac{d y_{a 2}}{d \tau} & =\frac{1}{3}\left\{x_{2}-\eta y_{a 2}-\gamma\left(y_{a 2}+y_{c 3}\right)\right\} \\
\frac{d y_{b 2}}{d \tau} & =\frac{1}{3}\left\{x_{2}-\eta y_{b 2}-\gamma\left(y_{b 2}+y_{b 4}\right)\right\} \\
\frac{d y_{c 2}}{d \tau} & =\frac{1}{3}\left\{x_{2}-\eta y_{c 2}-\gamma\left(y_{c 2}+y_{a 1}\right)\right\} .
\end{aligned}\right.
$$

[Third oscillator]

$$
\left\{\begin{aligned}
\frac{d x_{3}}{d \tau} & =\varepsilon\left(1-x_{3}{ }^{2}\right) x_{k}-\left(y_{a 3}+y_{b 3}+y_{c 3}\right) \\
\frac{d y_{a 3}}{d \tau} & =\frac{1}{3}\left\{x_{3}-\eta y_{a 3}-\gamma\left(y_{a 3}+y_{c 4}\right)\right\} \\
\frac{d y_{b 3}}{d \tau} & =\frac{1}{3}\left\{x_{3}-\eta y_{b 3}-\gamma\left(y_{b 3}+y_{b 1}\right)\right\} \\
\frac{d y_{c 3}}{d \tau} & =\frac{1}{3}\left\{x_{3}-\eta y_{c 3}-\gamma\left(y_{c 3}+y_{a 2}\right)\right\} .
\end{aligned}\right.
$$

[Fourth oscillator]

$$
\left\{\begin{aligned}
\frac{d x_{4}}{d \tau} & =\varepsilon\left(1-x_{4}{ }^{2}\right) x_{k}-\left(y_{a 4}+y_{b 4}+y_{c 4}\right) \\
\frac{d y_{a 4}}{d \tau} & =\frac{1}{3}\left\{x_{4}-\eta y_{a 4}-\gamma\left(y_{a 4}+y_{c 1}\right)\right\} \\
\frac{d y_{b 4}}{d \tau} & =\frac{1}{3}\left\{x_{4}-\eta y_{b 4}-\gamma\left(y_{b 4}+y_{b 2}\right)\right\} \\
\frac{d y_{c 4}}{d \tau} & =\frac{1}{3}\left\{x_{4}-\eta y_{c 4}-\gamma\left(y_{c 4}+y_{a 3}\right)\right\}
\end{aligned}\right.
$$

We use the following normalizations:

$$
\begin{gathered}
t=\sqrt{L C} \tau, v_{k}=\sqrt{\frac{g_{1}}{g_{3}}} x_{k}, i_{a k}=\sqrt{\frac{g_{1} C}{g_{3} L}} y_{a k}, \\
i_{b k}=\sqrt{\frac{g_{1} C}{g_{3} L}} y_{b k}, i_{c k}=\sqrt{\frac{g_{1} C}{g_{3} L}} y_{c k}, \\
\varepsilon=g_{1} \sqrt{\frac{L}{C}}, \gamma=R \sqrt{\frac{C}{L}}, \eta=r_{m} \sqrt{\frac{C}{L}}
\end{gathered}
$$

$(\mathrm{k}=1,2,3,4)$,

where $\varepsilon$ is the degree of nonlinearity, $\gamma$ is the coupling strength, and $\eta$ indicates the resistive component. In the computer simulations, we investigate the phase differences between adjacent oscillators.

\section{SYNCHRONIZATION PHENOMENA}

We calculate Eqs. (2)-(5) using a fourth-order Runge-Kutta method with the step size $h=0.02$ in this circuit. In this case, the parameters are set to $\varepsilon=0.50, \eta=0.02$ and $\gamma=0.15$. We exhibit the attractor of each oscillator in this simulation in Fig 4. In this figure, we show the horizontal axis is the voltage

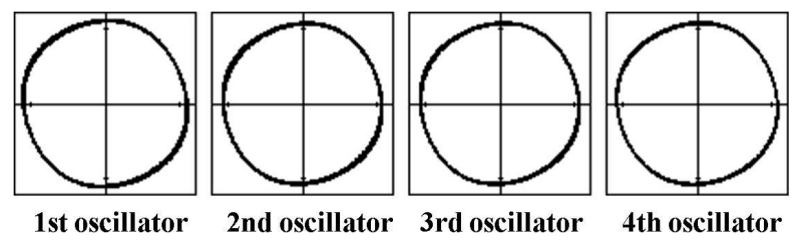

Fig. 4. Attractor of each oscillator. 

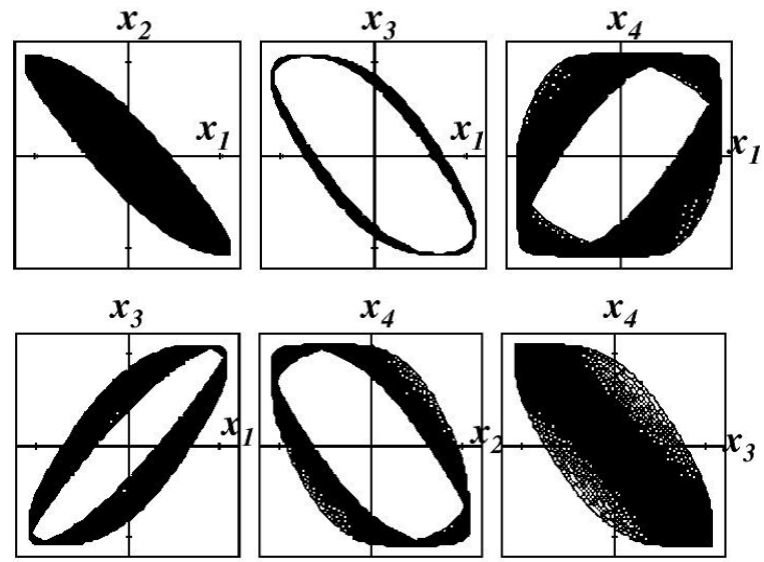

Fig. 5. Lissajous figures.

of each condenser, and the vertical axis is the electric current of each oscillator.

Next, the phase differences between the adjacent oscillators in all combinations at a certain time are shown in Fig. 5. We confirm that the rotation of phase difference is observed. Namely, the adjacent oscillators are trapped into the antiphase state at the certain time and are disconnected the synchronization periodically.

In order to investigate the mechanism of such synchronization phenomena in detail, the phase differences are calculated as shown in Fig. 6. The parameters are fixed as $\varepsilon=0.50$, $\eta=0.02$ and $\gamma=0.15$. In Fig. 6, "1st-2nd" indicates a
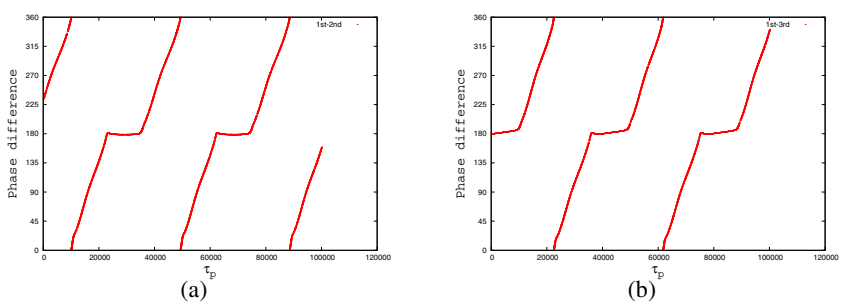

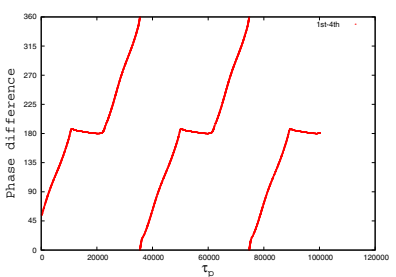

(c)

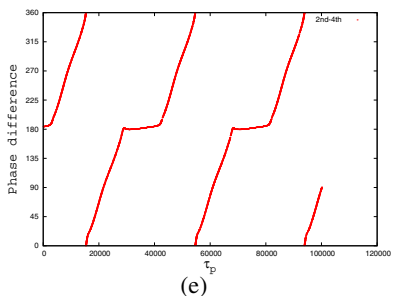

(e)

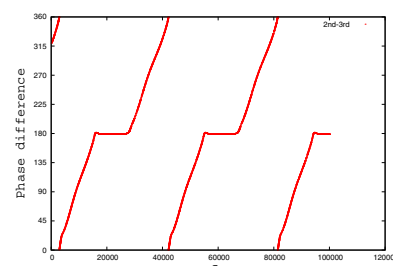

(d)

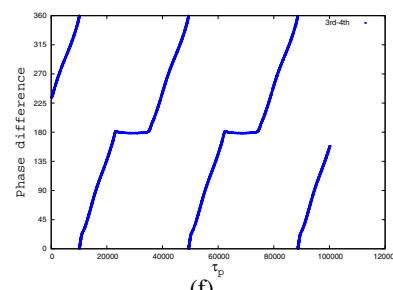

(f)
Fig. 6. The phase differences between adjacent oscillators. (a) 1st-2nd. (b) 1 st-3rd. (c) 2 nd-4th. (d) 2 nd-3rd. (e) 2 nd-4th. (f) 3 rd-4th. phase difference between the first oscillator and the second oscillator. The horizontal axis is the computer simulation time and the vertical axis is the phase difference. From this figure, we can see that the phase difference changes from $0^{\circ}$ to $360^{\circ}$ periodically. The coupled oscillators can not maintain the synchronization state and the phase differences change periodically after the adjacent oscillators are synchronized with the anti-phase states temporarily. The phase difference of all adjacent oscillators has a similar characteristic. Namely we find that the synchronization is gotten out from anti-phase and it becomes asynchronous.

\section{Sojourn Time of Anti-Phase State}

In this section, we measure the one period length of the changing phase difference and the sojourn time of the antiphase. We define the anti-phase state when the phase difference value is from $175^{\circ}$ to $185^{\circ}$. Figure 7 shows the definition of the one period and sojourn time of the anti-phase.

For this simulation, the iteration is set to $\tau_{p}=1,000,000$ for calculating the result more precisely. Figure 8 shows the simulation result of one period length and the sojourn time of the anti-phase state when the parameter of nonlinearity is changed from 0.10 to .200 . In this figure, we show the horizontal axis is $\varepsilon$, and the vertical axis is the length of one period and the sojourn time of the anti-phase state. We confirm that the length of one period and the sojourn time of the antiphase state increase with the nonlinearity $\varepsilon$.

Besides, we show the rate of the anti-phase state in Fig 9. In Fig 9, the ratio of the anti-phase state does not change so much with the nonlinearity $\varepsilon$.

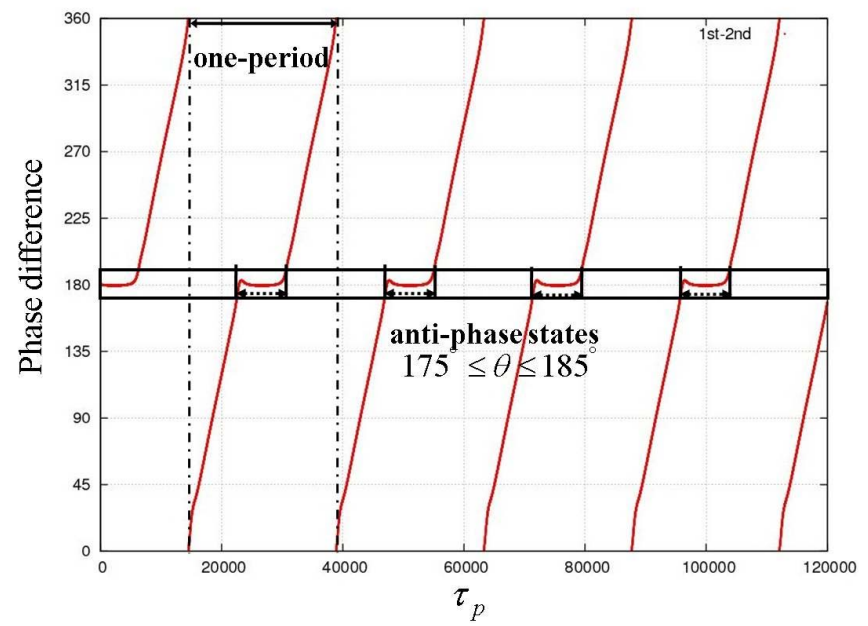

Fig. 7. Definition of the length of one-period and sojourn time of the antiphase.

\section{PoincARe MaPS}

Poincare map is one of the important methods to understand the structure of obits in the topological space. The one dimension of the dynamics system is reduced by using Poincare map. Figure 10 shows the simulation results of the Poincare map, when the nonlinearity $\varepsilon$ is fixed as $0.10,0.70$, 


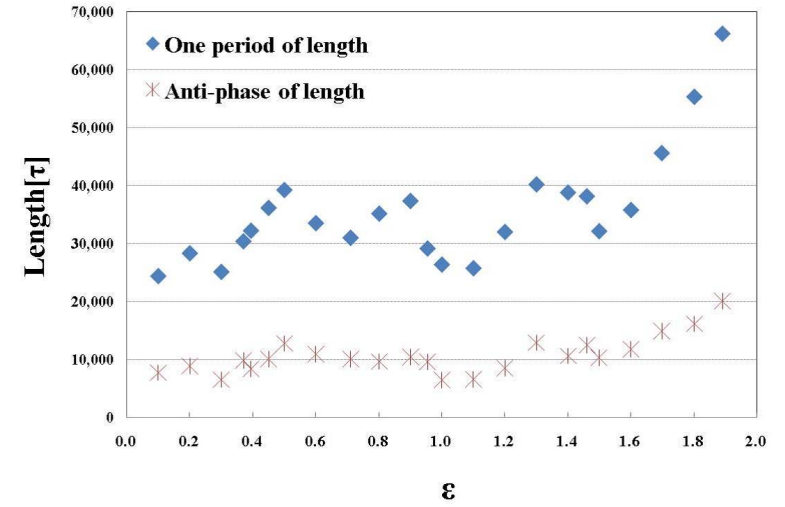

Fig. 8. Length of the one-period and sojourn time of the anti-phase.

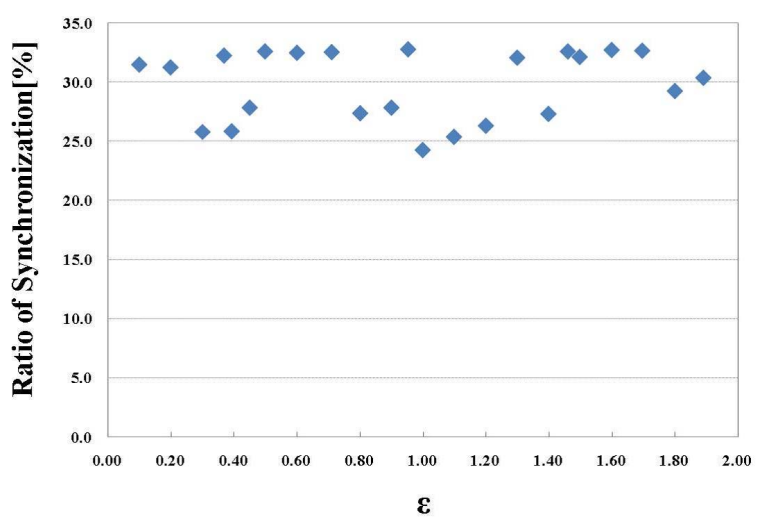

Fig. 9. Ratio of synchronization with anti-phase.

and 1.60. In the case of the left side figures (Fig. 10 (a), (c) and (e)), the horizontal axis denotes $y_{2}$. While, for the right side figures (Fig. 10 (b), (d) and (f)), the horizontal axis denotes $d x_{2} / d \tau$. From these figures, we can see that nonlinearity of the trajectory of Poincare map becomes strong with increasing the value of $\varepsilon$.

\section{CONCLUSION}

In this study, we have investigated synchronization phenomena observed in the four coupled oscillators as a regular tetrahedron form. In this circuit model, we observe the switching synchronization state between the adjacent oscillators. Namely, the coupled oscillators can not maintain the synchronization state and the phase differences change periodically after the adjacent oscillators are synchronized with the anti-phase states temporarily.

For the future work, we would like to investigate the relationship between the switching synchronization state observed in this study and the phase slip phenomena of noisily oscillatory systems.

\section{REFERENCES}

[1] L.L. Bonilla, C.J. Perez Vicente and R. Spigler, "Time-periodic phases in populations of nonlinearly coupled oscillators with bimodal frequency distributions," Physica D: Nonlinear Phenomena, vol.113, no.1, pp.7997, Feb. 1998

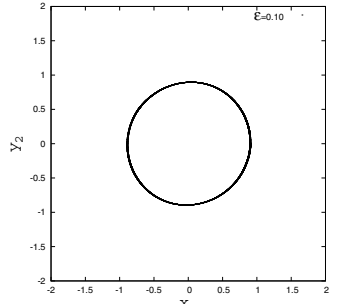

(a)

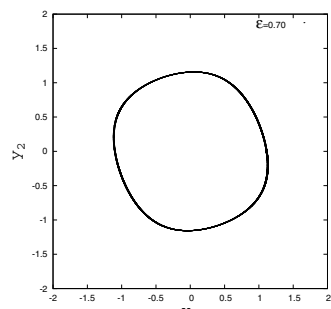

(c)

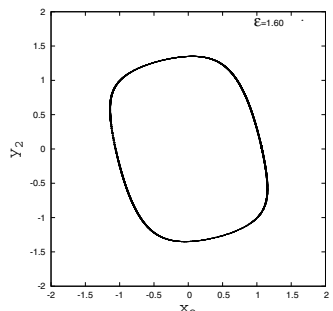

(e)

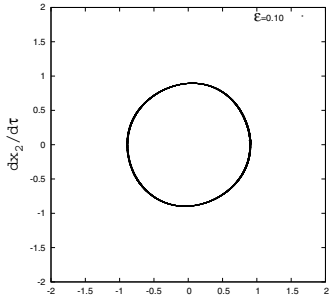

(b)

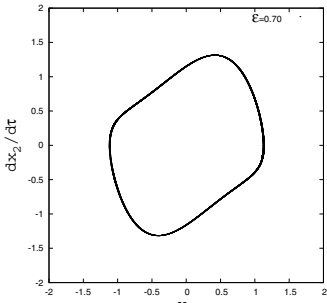

(d)

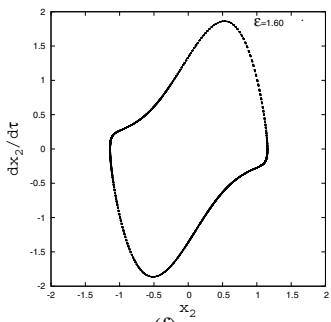

(f)
Fig. 10. Poincare map on the section $y_{a 1}=0$.(a) Horizontal: $x_{2}$, vertical: $y_{2}$ for the parameter $\varepsilon=0.10$. (b) Horizontal: $x_{2}$, vertical: $d x_{2} / d \tau$ for the parameter $\varepsilon=0.10$. (c) Horizontal: $x_{2}$, vertical: $y_{2}$ for the parameter $\varepsilon=0.70$. (d) Horizontal: $x_{2}$, vertical: $d x_{2} / d \tau$ for the parameter $\varepsilon=0.70$. (e) Horizontal: $x_{2}$, vertical: $y_{2}$ for the parameter $\varepsilon=1.60$. (f) Horizontal: $x_{2}$, vertical: $d x_{2} / d \tau$ for the parameter $\varepsilon=1.60$.

[2] J.A. Sherratt, "Invading wave fronts and their oscillatory wakes are linked by a modulated traveling phase resetting wave," Physica D: Nonlinear Phenomena, vol.117, no.1-4, pp.145-166, June 1998.

[3] G. Abramson, V.M. Kenkre and A.R. Bishop, "Analytic solutions for nonlinear waves in coupled reacting systems," Physica A: Statistical Mechanics and its Applications, vol.305, no.3-4, pp.427-436, Mar. 2002.

[4] 4. Belykh, M. Hasler, M. Lauret and H. Nijmeijer, "Synchronization and graph topology," International Journal of Bifurcation and Chaos, vol.15, no.11, pp.3423-3433, Nov. 2005.

[5] C.M. Gray, "Synchronous oscillations in neural systems: mechanisms and functions," J. Computational Neuroscience, vol.1, pp.11-38, 1994.

[6] R. Stoop and C. Wagner, "Neocortex's architecture optimizes computation, information transfer and synchronizability, at given total connection length," International Journal of Bifurcation and Chaos, vol.17, no.7, pp.2257-2279, 2007.

[7] T. Suezaki and S. Mori, "Mutual synchronization of two oscillators," Trans. IECE, vol.48, no.9, pp.1551-1557, Sep. 1965.

[8] T. Endo and S. Mori, "Mode analysis of a multimode ladder oscillator," IEEE trans. Circuit Syst., vol23, pp.100-113, Feb. 1976.

[9] T. Endo and S. Mori, "Mode analysis of two-dimensional low-pass multimode oscillator," IEEE trans. Circuit Syst., vol23, pp.517-530, Sep. 1976.

[10] T. Endo and S. Mori, "Mode analysis of a a large number of mutually coupled van der Pol oscillators," IEEE trans. Circuit Syst., vol25, pp.718, Feb. 1978.

[11] Y. Uwate, Y. Nishio and R Stoop, "Synchronization in three coupled van der Pol oscillators with different coupling Strength," Proc. of NCSP'10, pp.109-112, Mar. 2010 